\title{
Self-Similar Blow-Up Solutions of the KPZ Equation
}

\author{
Alexander Gladkov \\ Department of Mathematics and Mechanics, Belarusian State University, Nezavisimosti Avenue 4, 220030 Minsk, Belarus
}

Correspondence should be addressed to Alexander Gladkov; gladkoval@mail.ru

Received 7 July 2015; Accepted 16 August 2015

Academic Editor: Salim Messaoudi

Copyright (C) 2015 Alexander Gladkov. This is an open access article distributed under the Creative Commons Attribution License, which permits unrestricted use, distribution, and reproduction in any medium, provided the original work is properly cited.

Self-similar blow-up solutions for the generalized deterministic KPZ equation $u_{t}=u_{x x}+\left|u_{x}\right|^{q}$ with $q>2$ are considered. The asymptotic behavior of self-similar solutions is studied.

\section{Introduction}

We consider the generalized deterministic KPZ equation

$$
\frac{\partial u}{\partial t}=\frac{\partial^{2} u}{\partial x^{2}}+\left|\frac{\partial u}{\partial x}\right|^{q} \quad \text { for }(x, t) \in S_{T}:=\mathbb{R} \times(0, T)
$$

where $q>2$ and $T>0$. Equation (1) was first considered in the case $q=2$ by Kardar et al. [1] in connection with the study of the growth of surfaces. When $q=2$, (1) has since been referred to as the deterministic KPZ equation. For $q \neq 2$ it also called the generalized deterministic KPZ equation or Krug-Spohn equation because it was introduced in [2]. We refer to the review article [3] for references and a detailed historical account of the KPZ equation.

The existence and uniqueness of a classical solution of the Cauchy problem for (1) with $q=1$ and initial function $u_{0} \in C_{0}^{3}\left(\mathbb{R}^{n}\right)$ were proven in [4]. This result was extended to $u_{0} \in C^{2}\left(\mathbb{R}^{n}\right) \cap W^{2, \infty}\left(\mathbb{R}^{n}\right)$ and $q \geq 1$ in [5] and to $u_{0} \in$ $C\left(\mathbb{R}^{n}\right) \cap L^{\infty}\left(\mathbb{R}^{n}\right)$ and $q \geq 0$ in [6]. Several papers [7-11] were devoted to the investigation of the Cauchy problem for irregular initial data, namely, for $u_{0} \in L^{p}\left(\mathbb{R}^{n}\right), 1 \leq p<\infty$, or for bounded measures. The existence and uniqueness of a solution to the Cauchy problem with unbounded initial datum are proved in [12]. To confirm the optimality of obtained existence conditions, the authors of [12] analyze the asymptotic behavior of self-similar blow-up solutions of (1) for $q<2$.
In this paper we investigate the asymptotic behavior of self-similar blow-up solutions of (1) with $q>2$ having the form

$$
\begin{aligned}
u(x, t)=(T-t)^{\alpha} & f(\xi), \\
& \text { where } \xi=|x|(T-t)^{\beta}, 0<t<T .
\end{aligned}
$$

After substitution of (2) into (1) we find that

$$
\begin{aligned}
& \alpha=\frac{q-2}{2(q-1)}, \\
& \beta=-\frac{1}{2}
\end{aligned}
$$

and $f$ should satisfy the following equation:

$$
f^{\prime \prime}+\left|f^{\prime}\right|^{q}-\frac{1}{2} \xi f^{\prime}+\alpha f=0 \quad \text { on }(0,+\infty) .
$$

We will add to (4) the following initial data:

$$
\begin{aligned}
f(0) & =-f_{0}<0, \\
f^{\prime}(0) & =0 .
\end{aligned}
$$

Put

$$
C=\left[\frac{1}{q-1}\left(\frac{q-1}{q}\right)^{q}\right]^{1 /(q-1)} .
$$

Let us state the main result. 
Theorem 1. Let $u$ be a self-similar blow-up solution of (1) with $q>2$ which is defined in (2)-(5). Then

$$
\lim _{t \rightarrow T} u(x, t)(T-t)^{1 /(q-1)}=C|x|^{q /(q-1)} .
$$

A simple computation shows that Theorem 1 is a consequence of the following statement.

Theorem 2. Let $q>2$ and let $f$ be a solution of problem (4), (5). Then

$$
\lim _{\xi \rightarrow \infty} \frac{f(\xi)}{\xi q /(q-1)}=C
$$

The behavior of self-similar solutions for (1) of the type $u(x, t)=t^{\alpha} g\left(x t^{\beta}\right)$ has been analyzed in [13].

\section{The Proof of Theorem 2}

We start with a simple result which is used later on.

Lemma 3. Let $f$ be a solution of problem (4), (5) defined on $[0, \bar{\xi})$. Then

$$
\begin{gathered}
f^{\prime}(\xi)>0, \\
f^{\prime \prime}(\xi)>0
\end{gathered}
$$

$$
\text { for } \xi \in(0, \bar{\xi})
$$

Proof. Obviously, $f^{\prime \prime}(0)=\alpha f_{0}>0$. Therefore, by continuity, $f^{\prime \prime}>0$ and $f^{\prime}>0$ in some right-neighborhood of 0 . Suppose that there exists $\xi_{0}$ such that $0<\xi_{0}<\bar{\xi}, f^{\prime \prime}>0$ on $\left[0, \xi_{0}\right)$ and $f^{\prime \prime}\left(\xi_{0}\right)=0$. Then $f^{\prime}>0$ on $\left(0, \xi_{0}\right]$ and $f^{\prime \prime \prime}\left(\xi_{0}\right) \leq 0$. From (4) we find that $f^{\prime \prime \prime}\left(\xi_{0}\right)=f^{\prime}\left(\xi_{0}\right) /[2(q-1)]>0$. This contradiction proves (9).

Now we will obtain the upper bound for $f^{\prime}$.

Lemma 4. There exists $\xi_{0}>0$ such that

$$
f^{\prime}(\xi)<\left\{\frac{\xi}{2}\right\}^{1 /(q-1)} \quad \text { for } \xi \geq \xi_{0} .
$$

Proof. Lemma 3 implies that $f(\xi) \rightarrow \infty$ as $\xi \rightarrow \bar{\xi}$ and that there exists unique point $\xi_{0} \in(0, \bar{\xi})$ such that $f<0$ on $\left(0, \xi_{0}\right)$ and $f>0$ on $\left(\xi_{0}, \bar{\xi}\right)$. Substituting $f^{\prime \prime}>0$ and $f \geq 0$ in (4) yields $f^{\prime}(\xi)<\{\xi / 2\}^{1 /(q-1)}$ for $\xi \in\left[\xi_{0}, \bar{\xi}\right)$. Thus, $\bar{\xi}=\infty$ and (10) holds.

Changing variables in (4)

$$
f^{\prime}(\xi)=\xi^{1 /(q-1)} g(t), \quad \xi=\exp t,
$$

we get the new equation

$$
\begin{aligned}
g^{\prime \prime} & +\frac{3-q}{q-1} g^{\prime}-\frac{q-2}{(q-1)^{2}} g \\
& =\left\{\frac{1}{2} g^{\prime}-\left(g^{q}\right)^{\prime}+\frac{1}{q-1} g-\frac{q}{q-1} g^{q}\right\} \exp (2 t) .
\end{aligned}
$$

By (9), (10), and (11), there hold

$$
\begin{aligned}
& g(t)>0 \quad \text { for any } t \in \mathbb{R}, \\
& g(t)<\left\{\frac{1}{2}\right\}^{1 /(q-1)}, \\
& g^{\prime}(t)>-\frac{g}{q-1}
\end{aligned}
$$

for large values of $t$. Put

$$
\begin{aligned}
& C_{0}=\left\{\frac{1}{q}\right\}^{1 /(q-1)}, \\
& C_{1}=\left\{\frac{1}{2 q}\right\}^{1 /(q-1)} .
\end{aligned}
$$

It is obvious that $C_{0}>C_{1}$. Now we will establish the asymptotic behavior of $g(t)$ as $t \rightarrow+\infty$.

Lemma 5. Assume that $g$ is defined in (11). Then

$$
\lim _{t \rightarrow+\infty} g(t)=C_{0} .
$$

Proof. From a careful inspection of (12) we conclude that a local maximum of $g(t)$ can happen only when $g(t)>C_{0}$.

At first we suppose that $g(t)$ does not tend to $C_{0}$ as $t \rightarrow$ $+\infty$ and $g(t)$ is monotonic solution of (12) for large values of $t$. Then there exists $\bar{C} \neq C_{0}$ such that $\lim _{t \rightarrow \infty} g(t)=\bar{C}$. It is not difficult to show that for any $\varepsilon>0$ there exist $A>0$ and a sequence $\left\{t_{k}\right\}_{k=1}^{\infty}$ with the properties:

$$
\begin{gathered}
\lim _{k \rightarrow \infty} t_{k}=+\infty \\
\left|g^{\prime \prime}\left(t_{k}\right)\right| \leq A \\
\left|g^{\prime}\left(t_{k}\right)\right| \leq \varepsilon
\end{gathered}
$$

Indeed, let $g^{\prime} \geq 0$ for the definiteness. We suppose that $g^{\prime}(t)$ is not monotonic function for large values of $t$ since otherwise (17) is obvious. Denote by $\left\{\tau_{k}\right\}_{k=1}^{\infty}$ a sequence of local minima for $g^{\prime}$. Then (17) holds for some subsequence of $\left\{\tau_{k}\right\}_{k=1}^{\infty}$.

Passing to the limit in (12) as $t=t_{k} \rightarrow+\infty$ and choosing $\varepsilon$ in a suitable way we get that the left-hand side is bounded, while the right-hand side tends to infinity if $\bar{C} \neq 0$. Let $\bar{C}=0$. Using (13) and (14) we conclude from (12) that

$$
g^{\prime \prime}+\frac{3-q}{q-1} g^{\prime} \geq \frac{g}{3(q-1)} \exp (2 t)
$$

for large values of $t$. Then for large values of $k$ (17) and (18) imply

$$
g\left(t_{k}\right) \leq \gamma \exp \left(-2 t_{k}\right)
$$

where positive constant $\gamma$ does not depend on $k$. Setting $\xi_{k}=$ $\operatorname{expt}_{k}$, from (11) and (19), we get

$$
f^{\prime}\left(\xi_{k}\right) \leq \gamma \xi_{k}^{(3-2 q) /(q-1)}
$$

that contradicts (9). 
Now until the end of the proof we assume that $g(t)$ is not monotonic solution of (12) for large values of $t$. Suppose that $\liminf _{t \rightarrow \infty} g(t)<C_{0}$. Then there exist positive unbounded increasing sequences $\left\{s_{k}\right\}_{k=1}^{\infty}$ and $\left\{t_{k}\right\}_{k=1}^{\infty}$ such that $t_{k}>s_{k}$,

$$
g^{\prime}(t) \leq 0 \quad \text { for } t \in\left[s_{k}, t_{k}\right],
$$

and $g\left(s_{k}\right)=C_{0}, g\left(t_{k}\right)=C_{\star}$, where $C_{1}<C_{\star}<C_{0}$. Then

$$
\begin{aligned}
\frac{1}{2} g^{\prime}-\left(g^{q}\right)^{\prime} & =-q\left(g^{q-1}-C_{1}^{q-1}\right) g^{\prime} \\
& \geq-q\left(C_{\star}^{q-1}-C_{1}^{q-1}\right) g^{\prime} \geq 0 \quad \text { on }\left[s_{k}, t_{k}\right] .
\end{aligned}
$$

So, (12) and (22) imply that

$$
\begin{aligned}
& g^{\prime \prime}(t)+\frac{3-q}{q-1} g^{\prime}(t) \\
& \quad \geq-q\left(C_{\star}^{q-1}-C_{1}^{q-1}\right) g^{\prime}(t) \exp \left(2 s_{k}\right) \\
& \qquad \text { for } t \in\left[s_{k}, t_{k}\right] .
\end{aligned}
$$

Hence, integrating with respect to $t$ from $s_{k}$ to $t_{k}$, we get

$$
\begin{aligned}
& \left.\left\{g^{\prime}(t)+\frac{3-q}{q-1} g(t)\right\}\right|_{s_{k}} ^{t_{k}} \\
& \quad \geq q\left(C_{\star}^{q-1}-C_{1}^{q-1}\right)\left(C_{0}-C_{\star}\right) \exp \left(2 s_{k}\right) .
\end{aligned}
$$

This leads to a contradiction, since (13), (14), and (21) imply that the left-hand side of the last inequality is bounded, while the right-hand side becomes unbounded as $k \rightarrow \infty$.

Let us prove that $\liminf _{t \rightarrow \infty} g(t)=C_{0}$. Indeed, otherwise, there exist $\varepsilon>0$ and a sequence $\left\{\tau_{k}\right\}_{k=1}^{\infty}$ of local minima for $g$ with the properties $\tau_{k} \rightarrow+\infty$ as $k \rightarrow+\infty$ and

$$
g\left(\tau_{k}\right) \geq C_{0}+\varepsilon
$$

Passing in (12) to the limit as $t=\tau_{k} \rightarrow+\infty$ we get a contradiction.

To end the proof we show that $\limsup _{t \rightarrow \infty} g(t)=$ $C_{0}$. Otherwise, $\limsup _{t \rightarrow \infty} g(t)>C_{0}$. Then there exist unbounded increasing sequences $\left\{s_{k}\right\}_{k=1}^{\infty}$ and $\left\{t_{k}\right\}_{k=1}^{\infty}$ such that $t_{k}>s_{k}>2$,

$$
\begin{aligned}
g^{\prime}\left(s_{k}\right) & =0, \\
g^{\prime}\left(t_{k}\right) & =0, \\
g^{\prime}(t) & \geq 0 \\
g\left(t_{k}\right) & >C_{0}+\delta, \\
\left|g\left(s_{k}\right)-C_{0}\right| & <\varepsilon,
\end{aligned}
$$

for $t \in\left[s_{k}, t_{k}\right]$,

where $\delta>0$ and

$$
\varepsilon=\min \left\{\frac{\delta}{2}, \frac{q-1}{4 C_{0}} \delta^{2},\left[1-\left(\frac{7}{8}\right)^{1 /(q-1)}\right] C_{0}\right\} .
$$

Without loss of a generality we can suppose

$$
C_{0}-\varepsilon<g\left(s_{k}\right)<C_{0}
$$

or

$$
C_{0} \leq g\left(s_{k}\right)<C_{0}+\varepsilon .
$$

Let (28) be valid. If (29) is realized, the arguments are similar and simpler. Denote by $\left\{\bar{t}_{k}\right\}_{k=1}^{\infty}$ a sequence such that

$$
\begin{aligned}
\bar{t}_{k} & \in\left(s_{k}, t_{k}\right), \\
g\left(\bar{t}_{k}\right) & =C_{0} .
\end{aligned}
$$

Applying Hölder's inequality we derive

$$
\begin{aligned}
\int_{\bar{t}_{k}}^{t_{k}} g^{\prime}(\tau) d \tau \leq & \left(\int_{\bar{t}_{k}}^{t_{k}}\left(g^{\prime}(\tau)\right)^{2} \exp (2 \tau) d \tau\right)^{1 / 2} \\
& \cdot\left(\int_{\bar{t}_{k}}^{t_{k}} \exp (-2 \tau) d \tau\right)^{1 / 2}
\end{aligned}
$$

and therefore

$$
\int_{\bar{t}_{k}}^{t_{k}}\left(g^{\prime}(\tau)\right)^{2} \exp (2 \tau) d \tau \geq 2 \delta^{2} \exp \left(2 \bar{t}_{k}\right)
$$

We multiply (12) by $g^{\prime}(t)$ and integrate after over $\left[s_{k}, t_{k}\right]$. Using (15), (26)-(28), (30), and (32) we obtain

$$
\begin{aligned}
& -\frac{q-2}{2(q-1)^{2}} g^{2}\left(t_{k}\right) \leq \frac{q-3}{q-1} \int_{s_{k}}^{t_{k}}\left(g^{\prime}(\tau)\right)^{2} d \tau \\
& +\int_{s_{k}}^{t_{k}}\left(g^{\prime}(\tau)\right)^{2}\left[\frac{1}{2}-q g^{q-1}(\tau)\right] \exp (2 \tau) d \tau \\
& +\frac{\exp \left(2 \bar{t}_{k}\right)}{q-1} \\
& \cdot \int_{s_{k}}^{\bar{t}_{k}}\left[\frac{1}{2}\left(g^{2}(\tau)\right)^{\prime}-\frac{q}{q+1}\left(g^{q+1}(\tau)\right)^{\prime}\right] d \tau \leq-\frac{1}{4} \\
& \cdot \int_{\bar{t}_{k}}^{t_{k}}\left(g^{\prime}(\tau)\right)^{2} \exp (2 \tau) d \tau \\
& +\left.\frac{\exp \left(2 \bar{t}_{k}\right)}{q-1}\left(\frac{g^{2}(\tau)}{2}-\frac{q g^{q+1}(\tau)}{q+1}\right)\right|_{s_{k}} ^{\bar{t}_{k}} \\
& \quad \leq\left[-\frac{\delta^{2}}{2}+\frac{\varepsilon C_{0}}{q-1}\right] \exp \left(2 \bar{t}_{k}\right) \leq-\frac{\delta^{2}}{4} \exp \left(2 \bar{t}_{k}\right) .
\end{aligned}
$$

Passing to the limit as $k \rightarrow \infty$ we get a contradiction with (14).

Now (8) is a simple consequence of Lemma 5 and the definition of $g(t)$.

Remark 6. Note that Theorem 2 demonstrates the optimality of Theorem 2.3 in [12]. The arguments are the same as in Remark 4.6 of that paper. 
Our next result shows that (4) with initial data

$$
\begin{aligned}
f(0) & =f_{0}>0, \\
f^{\prime}(0) & =0
\end{aligned}
$$

has no global solution.

Theorem 7. Let $q>2$ and let $f$ be a solution of problem (4), (34). Then there exists $\xi_{\star}$ such that $0<\xi_{\star}<+\infty$ and $f(\xi) \rightarrow$ $-\infty$ as $\xi \uparrow \xi_{\star}$.

Proof. Suppose that problem (4), (34) has a solution $f$ that is infinitely extendible to the right. Using the arguments of Lemma 3 we show that $f^{\prime}<0$ and $f^{\prime \prime}<0$ on $(0,+\infty)$. From (4) we obtain

$$
f^{\prime \prime \prime}(\xi)<-\left(\left|f^{\prime}(\xi)\right|^{q}\right)^{\prime} .
$$

After the integration of (35) over $[0, \xi]$ we conclude that

$$
f^{\prime \prime}(\xi)<-\left|f^{\prime}(\xi)\right|^{q} .
$$

Integrating (36) over $\left[\xi_{1}, \xi\right]\left(0<\xi_{1}<\xi\right)$ we infer

$$
\frac{1}{(q-1)\left|f^{\prime}\left(\xi_{1}\right)\right|^{q-1}}>\xi-\xi_{1} \text {. }
$$

Passing to the limit as $\xi \rightarrow \infty$ we obtain a contradiction which proves the theorem.

\section{Conflict of Interests}

The author declares that there is no conflict of interests regarding the publication of this paper.

\section{Acknowledgment}

This work was supported by the State Research Program of Belarus (Grant no. 1.2.03).

\section{References}

[1] M. Kardar, G. Parisi, and Y.-C. Zhang, "Dynamic scaling of growing interfaces," Physical Review Letters, vol. 56, no. 9, pp. 889-892, 1986.

[2] J. Krug and H. Spohn, "Kinetic roughening of growing surfaces," in Solids Far from Equilibrium, C. Godrèche, Ed., pp. 479-582, Cambridge University Press, Cambridge, UK, 1991.

[3] I. Corwin, "The Kardar-Parisi-Zhang equation and universality class," Random Matrices: Theory and Applications, vol. 1, Article ID 1130001, p. 76, 2012.

[4] M. Ben-Artzi, "Global existence and decay for a nonlinear parabolic equation," Nonlinear Analysis, vol. 19, no. 8, pp. 763$768,1992$.

[5] L. Amour and M. Ben-Artzi, "Global existence and decay for viscous Hamilton-Jacobi equations," Nonlinear Analysis, Theory, Methods and Applications, vol. 31, no. 5-6, pp. 621-628, 1998.

[6] B. H. Gilding, M. Guedda, and R. Kersner, "The Cauchy problem for $u_{t}=\Delta u+|\nabla u|^{q}$," Journal of Mathematical Analysis and Applications, vol. 284, no. 2, pp. 733-755, 2003.
[7] S. Benachour and P. Laurençot, "Global solutions to viscous Hamilton-Jacobi equations with irregular initial data," Communications in Partial Differential Equations, vol. 24, no. 11-12, pp. 1999-2021, 1999.

[8] S. Benachour, B. Roynette, and P. Vallois, "Solutions fondamentales de $u_{t}-1 / 2 u_{x x}= \pm\left|u_{x}\right|$," Astérisque, vol. 236, pp. 41-71, 1996.

[9] M. Ben-Artzi, J. Goodman, and A. Levy, "Remarks on a nonlinear parabolic equation," Transactions of the American Mathematical Society, vol. 352, no. 8, pp. 731-751, 2000.

[10] M. Ben-Artzi, P. Souplet, and F. B. Weissler, "Sur la nonexistence et la non-unicité des solutions du problème de Cauchy pour une équation parabolique semi-linéaire," Comptes Rendus de l'Académie des Sciences Series I: Mathematics, vol. 329, no. 5, pp. 371-376, 1999.

[11] M. Ben-Artzi, P. Souplet, and F. B. Weissler, "The local theory for viscous hamilton-Jacobi equations in Lebesgue spaces," Journal des Mathematiques Pures et Appliquees, vol. 81, no. 4, pp. 343378, 2002.

[12] A. Gladkov, M. Guedda, and R. Kersner, "A KPZ growth model with possibly unbounded data: correctness and blowup," Nonlinear Analysis, Theory, Methods and Applications, vol. 68, no. 7, pp. 2079-2091, 2008.

[13] M. Guedda and R. Kersner, "Self-similar solutions to the generalized deterministic KPZ equation," Nonlinear Differential Equations and Applications, vol. 10, no. 1, pp. 1-13, 2003. 


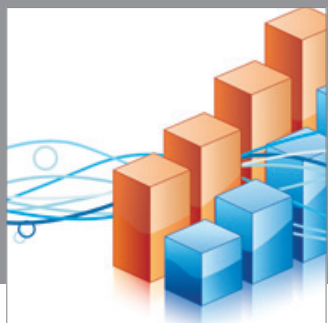

Advances in

Operations Research

mansans

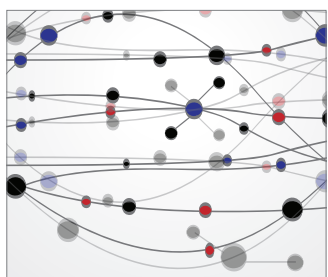

The Scientific World Journal
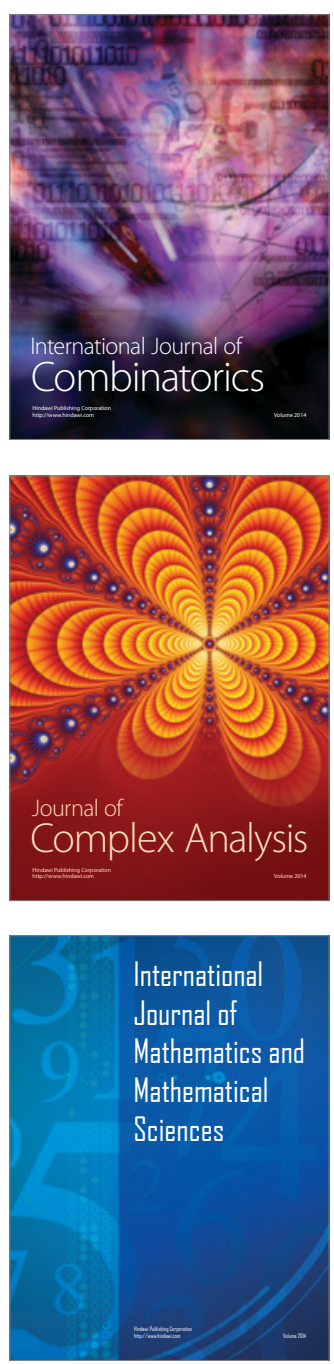
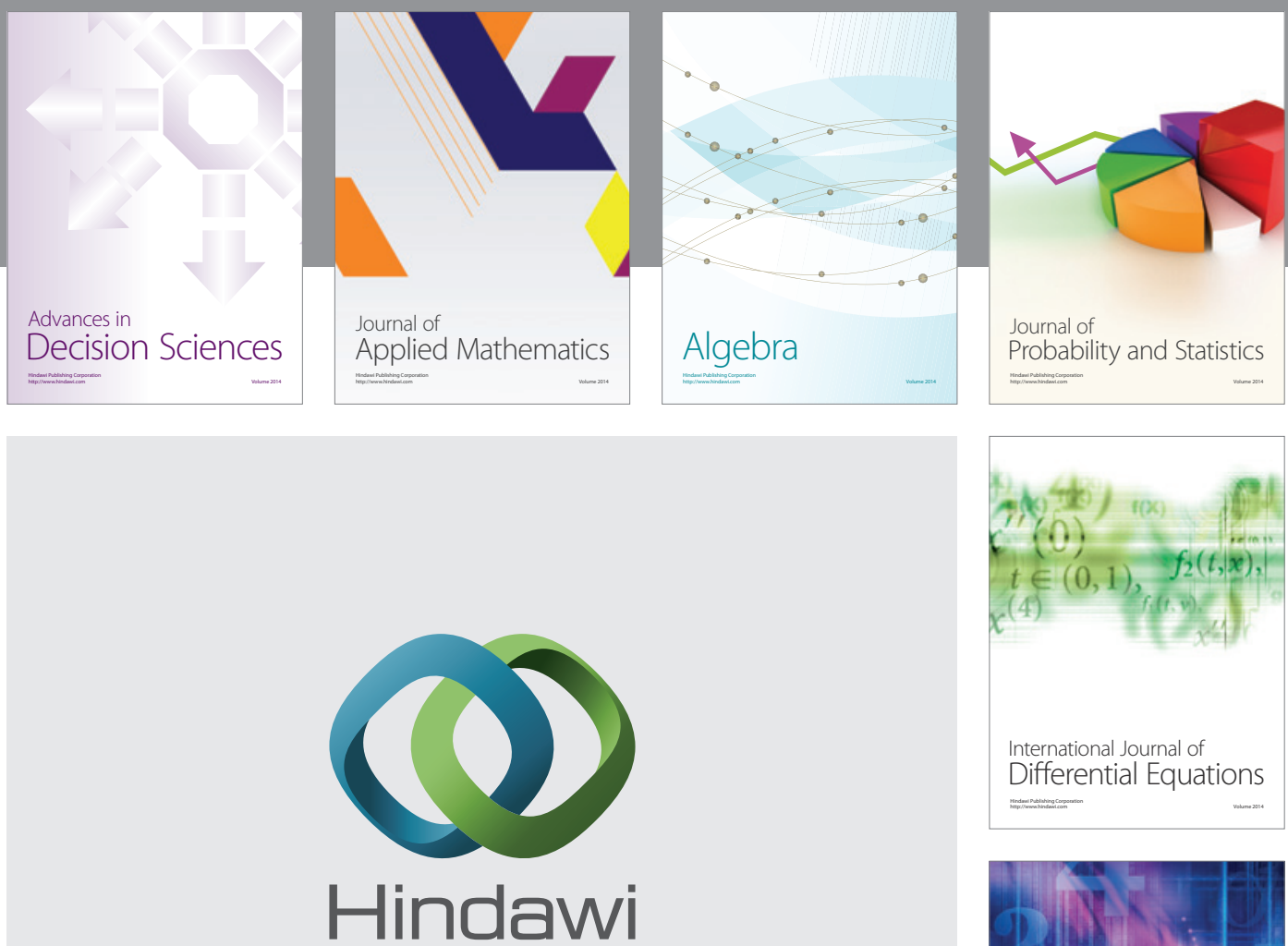

Submit your manuscripts at http://www.hindawi.com
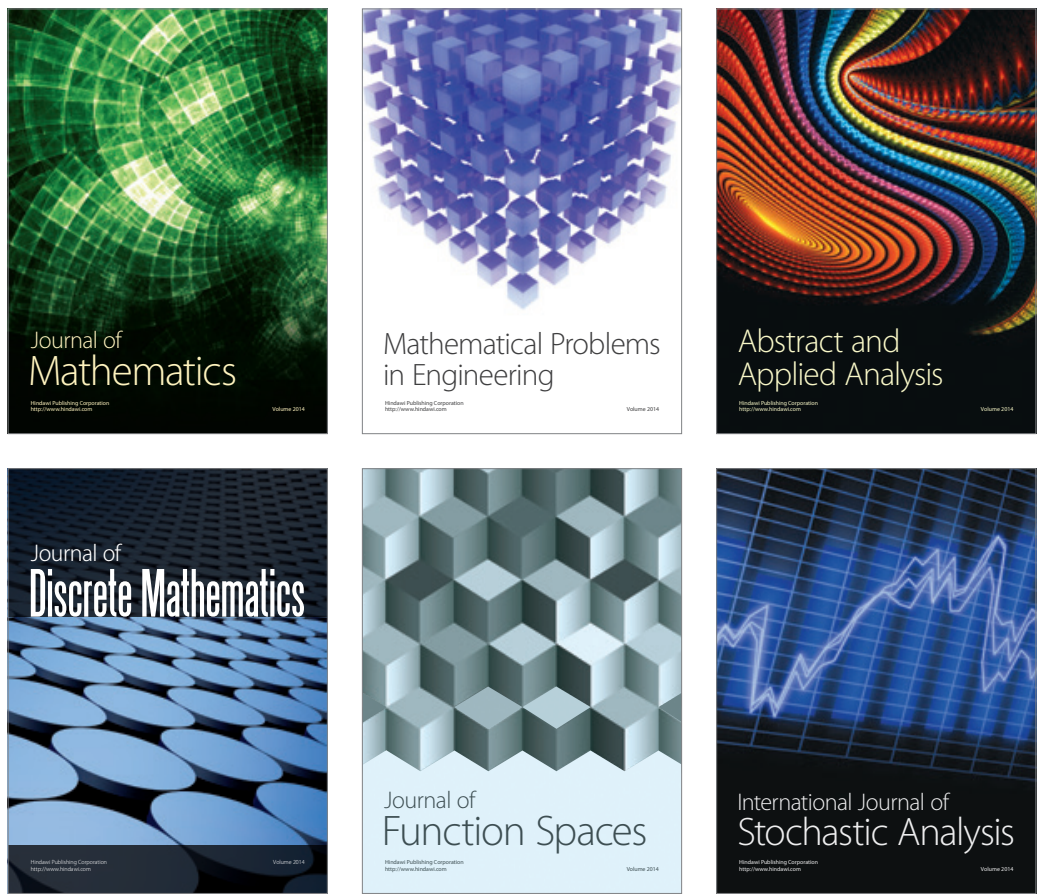

Journal of

Function Spaces

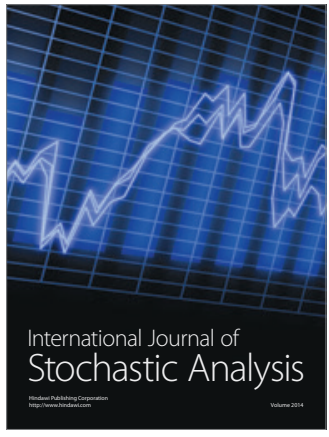

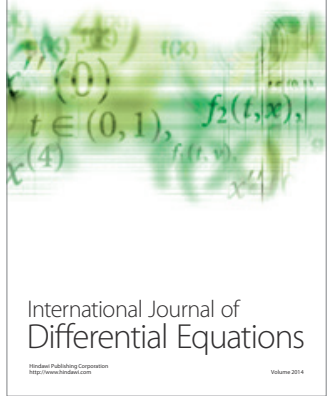
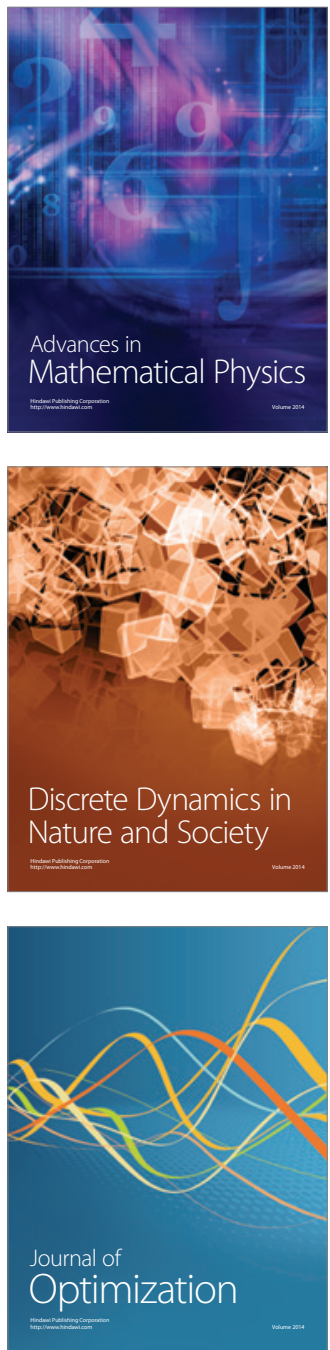